\title{
Une histoire de silences
}

\author{
Alexandre Vincent
}

La parution récente de l'Histoire du silence d'Alain Corbin a mis sous les feux de l'actualité éditoriale une catégorie sonore peu fréquemment à l'honneur ${ }^{1}$. Avec ce livre, le silence semblait trouver son historien, un auteur dont la longue carrière fut marquée par l'aptitude à faire émerger de nouveaux objets de recherche, dans la veine d'une « histoire des sensibilités » chère à Lucien Febvre et Robert Mandrou ${ }^{2}$. L'historiographie anglophone reconnaît volontiers la grande influence de celui qui accepta, en 2000, le qualificatif d'«historien du sensible» dans la naissance et le développement d'un champ désormais florissant: l'histoire des sens ${ }^{3}$. Bien

Sauf indications contraires, les textes latins sont cités dans la Collection des universités de France, Paris, Les Belles Lettres.

1 - Alain Corbin, Histoire du silence. De la Renaissance à nos jours, Paris, Albin Michel, 2016. 2 - Lucien FEbVRe, "La sensibilité et l'histoire», Annales d'histoire sociale, 3-3/4, 1941, p. 5-20; Robert MANDrou, « Pour une histoire des sensibilités », Annales ESC, 14-3, 1959, p. $581-588$.

3 - Alain Corbin, Historien du sensible. Entretiens avec Gilles Heuré, Paris, La Découverte, 2000. Sur la reconnaissance par l'historiographie anglophone de l'impulsion décisive d'A. Corbin dans l'histoire des sens, voir Mark M. SMITH, Sensory History, Oxford, Berg, 2007, p. 7 sq. ; Ari Y. Kelman, «Rethinking the Soundscape: A Critical Genealogy of a Key Term in Sound Studies", Senses and Society, 5-2, 2010, p. 212-234, ici p. 226-228; David Howes et Constance Classen, Ways of Sensing: Understanding the Senses in Society, New York, Routledge, 2014; ou encore la référence à ses travaux dans l'introduction de Jonathan STERNE, Une histoire de la modernité sonore, trad. par M. Boidy, Paris, La Découverte, [2003] 2015, p. 9 et 24. La traduction en anglais systématique des ouvrages d'A. Corbin est aussi significative qu'explicative de l'importance de sa réception outre-Atlantique. 
que l'existence d'un projet global cherchant à décliner des études sur les sens envisagés les uns après les autres soit réfutée par A. Corbin, l'évolution de sa bibliographie au cours des décennies 1980 et 1990 témoigne de l'extension de ses préoccupations, de l'odorat au toucher en passant par l'ouïe ${ }^{4}$, puis de la généralisation de son expertise aux essais de synthèse sur les acquis et les perspectives de ce champ en devenir qu'était alors la sensory history ${ }^{5}$. Partie intégrante, voire fondamentale, de l'environnement sonore, le silence constitue donc un objet d'étude situé dans la droite ligne des thématiques de l'auteur. Bien plus, une telle enquête a été annoncée dès 1995, quelques mois après la parution des Cloches de la terre, où il est question de s'interroger sur «la nature, les rythmes et les qualités du silence [...], toile de fond sur laquelle se détachent les bruits et les sons qu'il rend plus ou moins perceptibles ${ }^{6}$ ".

Derrière cette cohérence dans la démarche, l'Histoire du silence propose au lecteur une approche méthodologique qui contraste avec les objectifs fixés dans les ouvrages précédents. A. Corbin se revendique d'une histoire qui place le ressenti au-dessus de l'analyse: "Trop souvent l'histoire a prétendu expliquer. Quand elle aborde le monde des émotions, il lui faut aussi et surtout faire ressentir, en particulier quand les univers mentaux ont disparu.» L'outillage mis au service de cette ambition est simple, il s'agit de «citations révélatrices ${ }^{7}$. Dès lors, A. Corbin entrâne ses lecteurs dans des flâneries littéraires, au fil de sa grande connaissance des œuvres produites entre la fin du XVIII ${ }^{\mathrm{e}}$ et le premier $\mathrm{XX}^{\mathrm{e}}$ siècle ${ }^{8}$. Parfaitement respectable, une telle méthodologie visant à faire ressentir pourrait être interprétée comme une forme de revendication d'une inscription dans le sensual turn des sciences sociales, que l'anthropologue David Howes appelle

4 - Alain CORBIN, Le territoire du vide. L'Occident et le désir du rivage, 1750-1840, Paris, Aubier, 1988, dans lequel, bien que le toucher ne soit pas le principal sujet d'investigation, les sensations du soleil et de la mer sur la peau, ou du sable sous les pieds, sont en filigrane de l'enquête; Id., Le miasme et la jonquille. L'odorat et l'imaginaire social, XVIII'-XIX siècles, Paris, Flammarion, 1986; Id., Les cloches de la terre. Paysage sonore et culture sensible dans les campagnes au XIXe siècle, Paris, Aubier, 1990.

5 - Alain Corbin, «Histoire et anthropologie sensorielle» [1990], Le temps, le désir et l'horreur. Essais sur le dix-neuvième siècle, Paris, Aubier, 1991, p. 227-244; Id., "Charting the Cultural History of the Senses », in D. Howes (dir.), Empire of the Senses: The Sensual Culture Reader, Oxford, Berg, 2005, p. 128-139. Sur la défense d'une entreprise systématique d'étude fractionnée des sens, voir A. Corbin, Historien du sensible..., op. cit.

6 - Alain Corbin, "Prélude à une histoire de l'espace et du paysage sonores ", in M. Porret et F. Rosset (dir.), Le jardin de l'esprit. Textes offerts à Bronistaw Baczko, Genève, Droz, 1995, p. 51-63, ici p. 52. La même idée est répétée en des termes quasiment identiques dans Id., L'homme dans le paysage. Entretien avec Jean Lebrun, Paris, Textuel, 2001, p. 44: «Il n'est pas d'étude du paysage sonore sans réflexion sur le silence, puisque celui-ci constitue une toile de fond qui conditionne la possibilité de l'appréciation.»

7 - A. CORBIn, Histoire du silence..., op. cit., p. 9.

8 - Il s'agit des bornes réelles de l'enquête, qui ressortent de facto des sources mobilisées. La Renaissance tardive n'est que marginalement concernée dans le chapitre consacré aux quêtes spirituelles du silence. Le monde très contemporain est pour sa part totalement absent, n'était-ce deux lignes de l'introduction. 
avec force de ses vœux depuis de nombreuses années : il ne s'agit pas seulement d'adopter des sujets relatifs au sens, mais aussi de changer les modalités de production du monde académique, afin de les rendre elles-mêmes plus sensibles ${ }^{9}$. Le choix opéré par A. Corbin, que l'on discerne à travers la structure de son ouvrage, n'en est pas moins problématique en ce qu'il révèle des objectifs de l'historien visà-vis de son sujet : évoquer plutôt qu'expliquer? La question est particulièrement importante lorsqu'elle s'applique à un objet aussi volatile et difficilement cernable que le son du passé, et plus encore ses silences. Serait-on condamné sur ces thématiques à une invitation à la méditation?

En adoptant délibérément la position du flâneur sur un sujet lié aux perceptions sonores, A. Corbin appelle au rapprochement avec Raymond Murray Schafer, dont la présence hante la partie de son œuvre consacrée aux sons ${ }^{10}$. La notion de "paysage sonore ", énoncée par le musicologue, connut - et connaît encore - un succès extraordinaire auquel A. Corbin participa, à sa mesure. L'objet d'étude rapproche les deux hommes mais, plus encore ici, c'est leurs méthodes qui méritent d'être comparées. Évocation contre analyse, émotion contre sensation : la lecture critique de l'Histoire du silence appelle en réalité à une plongée méthodologique dans le champ de l'anthropologie historique du sens auditif. À l'opposé de l'approche d'A. Corbin présentée dans une première partie, le second temps de l'article propose une étude strictement contextualisée, limitée au seul domaine de la religion romaine. Le silence y est considéré non seulement comme une catégorie acoustique mais aussi comme un cadre contraignant de l'orthopraxie rituelle. Loin d'une évocation, il s'agit de comprendre les multiples fonctions du silence dans le cadre normé des rites de la religion publique.

\section{Une lecture critique de l'Histoire du silence}

\section{Attrait et répulsion du paysage sonore}

Malgré l'importance de sa production musicale, M. Schafer est avant tout l'homme d'une notion qui a fait florès: le soundscape ${ }^{11}$. Forgé à la toute fin des années 1960, le néologisme est traduit en français par l'expression "paysage sonore ${ }^{12}$ » dès 1979 , sous la plume de Sylvette Gleize. La création d'un terme permettant de désigner

9 - David Howes, Sensual Relations: Engaging the Senses in Culture and Social Theory, Ann Arbor, The University of Michigan Press, 2010, p. 29-58. Le conditionnel reste de rigueur car si A. Corbin connaît évidemment les travaux de D. Howes, il ne fait pas état d'une adhésion volontaire au sensual turn dans son livre.

10 - La tentation, voire la revendication, d'être un «historien flâneur » est déjà suggérée par G. Heuré et acceptée par A. CoRBIN, Historien du sensible..., op. cit., p. 65.

11 - Compositeur et musicologue, M. Schafer a par ailleurs laissé aux adeptes de cette discipline des outils de classification des sons encore utilisés aujourd'hui.

12 - Raymond Murray SCHAFER, The New Soundscape: A Handbook for the Modern Music Teacher, Toronto, Berandol Music, 1969. Avec cet opuscule, l'auteur souhaitait attirer l'attention des enseignants de musique sur la nécessité d'ouvrir le contenu de leurs cours aux bruits de la nature, menacés par le monde moderne. La notion de soundscape est 
de manière synthétique la complexité de l'organisation des perceptions sonores assura à la notion un succès fulgurant. Elle se situait dans une longue tradition de travaux engageant à décloisonner l'écoute de la musique pour l'ouvrir vers le son; elle s'intégrait également aux préoccupations écologiques naissantes et bénéficia, en outre, de l'efficacité poétique de la synesthésie induite par la concaténation en anglais des termes sound et landscape ${ }^{13}$. Quelques vingt-cinq années après la publication de l'ouvrage qui le popularisa, Helmi Järviluoma et Gregg Wagstaff tirèrent le bilan des soundscape studies, qu'ils repéraient alors autant dans l'architecture, les arts acoustiques, la communication, l'éducation ou la géographie que dans la musique, la physique, la psychologie, la radiophonie, la sociologie ou l'urbanisme ${ }^{14}$. À cette liste à la Prévert des disciplines s'ajoutent aisément la sociologie urbaine, l'anthropologie, la musicologie ou encore l'histoire. Derrière ce foisonnement se donne à lire un champ en émergence, celui d'une branche cousine des sound studies qui peine à se structurer, une réalité qui a peu évolué quinze ans plus $\operatorname{tard}{ }^{15}$. Les études qui se réfèrent plus ou moins directement au soundscape abondent, mais elles sont loin d'être uniformes quant à ce qu'elles entendent par la notion, comme le constate Ari Kelman ${ }^{16}$. Les raisons de cet éparpillement résident incontestablement dans un effet de mode lié au développement des études sur les sens de manière générale, comme avatars de l'histoire du corps: les sensory studies, dont les sound studies constituent, à leur manière, une catégorie ${ }^{17}$. Elles doivent être cherchées, par ailleurs, dans le faible niveau de définition fourni par l'inventeur d'une notion qui semble s'être imposée avant tout par habitude ${ }^{18}$. La signification du «paysage sonore» est donnée manifestement pour évidente : celui-ci serait

davantage développée dans Id., Le paysage sonore. Toute l'histoire de notre environnement sonore à travers les âges, trad. par S. Gleize, Paris, J.-C. Lattès, [1977] 1979. L'utilisation de la notion clé dans le titre français, contrairement à la publication originelle, est révélatrice de l'importance de sa réception.

13 - Sur les questions de réception du soundscape et sur l'utilisation de la notion dans les sciences sociales, voir Alexandre VINCENT, «Paysage sonore et sciences sociales: sonorités, sens, histoire », in S. EMERIT, S. PERROT et A. VinCENT (dir.), Le paysage sonore de l'Antiquité. Méthodologie, historiographie, perspectives, Le Caire, Presses de l'Institut français d'archéologie orientale, 2015, p. 9-40.

14 - Helmi Järviluoma et Gregg Wagstaff, «Soundscape Studies and Methods: An Introduction », in H. JÄrviluoma et G. WagSTAFF (dir.), Soundscape Studies and Methods, Helsinki, Finnish Society for Ethnomusicology, 2002, p. 9-26, ici p. 11.

15 - Michele Hilmes, «Is There a Field Called Sound Culture Studies? And Does It Matter? », American Quarterly, 57-1, 2005, p. 249-259.

16 - A. Y. Kelman, «Rethinking the Soundscape...», art. cit.

17 - Notamment à travers la notion de culture auditive: Michael BULL et Les BACK, «Introduction: Into Sound », in M. BuLL et L. BACK (dir.), The Auditory Culture Reader, New York, Berg, 2003, p. 1-18.

18 - Dans sa préface, Raymond Murray SCHAFER, Le paysage sonore. Le monde comme musique, trad. par S. Gleize, Marseille, Éd. Wildproject, [1977] 2010, p. 14, relate ainsi la naissance de la notion: «Il nous fallait un terme pour définir nos études, et c'est alors que le mot soundscape ('paysage sonore') est entré dans le vocabulaire. Il vient de landscape ('paysage'). Le landscape est, ni plus ni moins, tout ce qui peut être vu, ainsi le soundscape est-il devenu tout ce qui pouvait être entendu.» 
l'équivalent, selon M. Schafer, de l'«environnement des sons ${ }^{19}$ ». Il est ce que l'on peut saisir, par l'oreille ou par des moyens d'enregistrement, en un lieu et en un moment donnés.

Conscients des dangers qu'il y aurait à se revendiquer d'une notion trop faiblement définie et sujette à de multiples interprétations, particulièrement en français au vu du bagage historiographique charrié par le terme "paysage ${ }^{20}$ ", certains chercheur invitent à l'abandonner pour lui substituer la terminologie des « univers sonores ${ }^{21}$ ", des «ambiances soniques ${ }^{22}$ ", des «milieux sonores ${ }^{23}$ ", ou encore celle d' ' environnement sonore ${ }^{24}{ }$. Par-delà des divergences qui dépassent la simple querelle sémantique ${ }^{25}$, ces appels au changement respectent ce qui fait la spécificité du paysage sonore, c'est-à-dire son rapport à l'espace. Le principe du paysage sonore est celui d'une écoute localisée et ancrée dans un environnement qui lui est propre. Les sons qu'il est question d'étudier n'apparaissent pas ex nihilo. Ils ne sont objets de l'enquête qu'en tant que partie d'un tout qui ne saurait s'abstraire de sa localisation. Ce n'est pas la moindre vertu du paysage sonore que de rappeler cet ancrage contextuel de la production et de la perception acoustiques, une dimension parfois oubliée dans les études plus générales des sound studies.

S'il est impossible de résumer en quelques lignes l'ébullition intellectuelle qui caractérise cette variante des studies, tout comme l'importance de la production scientifique qui lui est consacrée, c'est à l'évidence le son, dans sa dimension physique, qui les unifie et non un quelconque contexte d'exécution ou de réception ${ }^{26}$. Les sound studies font la part belle aux médias et aux enregistrements qui

19 - Ibid., p. 384. Un glossaire d'« écologie sonore » accompagne l'ouvrage et développe la définition de la notion: "Techniquement, toute partie de cet environnement est prise comme champ d'étude. Le terme s'applique aussi bien à des environnements réels qu'à des constructions abstraites, tels que compositions musicales ou montages sur bande, en particulier lorsqu'ils sont considérés comme faisant partie du cadre de vie. »

20 - A. Vincent, «Paysage sonore et sciences sociales...», art. cit.; Christophe VENDRIES, «Du bruit dans la cité. L'invention du 'paysage sonore' et l'Antiquité romaine ", in S. EMERIT, S. PERrot et A. Vincent (dir.), Le paysage sonore..., op. cit., p. 209-258, ici p. 209-214.

21 - Olivier BALAŸ, L'espace sonore de la ville au XIX siècle, Paris, À la croisée, 2003.

22 - Grégoire ChelKofF, «L'homme sonore en contexte urbain. Les trente premières années du laboratoire Cresson », in J. CANDAU et M.-B. LE GONIDEC (dir.), Paysages sensoriels. Essai d'anthropologie de la construction et de la perception de l'environnement sonore, Paris, CThs, 2013, p. 177-198; Jean-Paul Thibaud, «Petite archéologie de la notion d'ambiance ", Communications, 90, 2012, p. 155-174.

23 - Christine Guillebaud (dir.), Toward an Anthropology of Ambient Sound, Londres, Routledge, 2017. L'ouvrage est le produit d'un groupe de recherche dénommé MILSON «Pour une anthropologie des milieux sonores».

24 - J. Candau et M.-B. Le Gonidec (dir.), Paysages sensoriels..., op. cit. Les auteurs acceptent aussi «ambiance sonore», «milieu sonore» ou «environnement acoustique». 25 - Tim Ingold, « Against Soundscape », in A. CarlyLE (dir.), Autumn Leaves: Sound and the Environment in Artistic Practice, Paris, Double entendre/CrISAP, 2007, p. 10-13.

26 - Jonathan STERne, «Sonic Imaginations", in J. STERne (dir.), The Sound Studies Reader, Londres, Routledge, 2012, p. 1-17. Un livre incarne à lui seul le foisonnement baroque des sound studies: Hillel SCHWARTZ, Making Noise: From Babel to the Big Bang and beyond, New York, Zone Books, 2011. Pour un bilan récent, voir Bruce JoHnSON, « Sound 
distinguent structurellement les contextes de production et d'écoute. Les «sound students ", pour reprendre l'expression de Jonathan Sterne, se focalisent en effet sur la période de diffusion des moyens de conservation et de reproduction de sons, considérée comme un moment historique de rupture ${ }^{27}$. La mise au point des technologies d'enregistrement à la fin du XIX ${ }^{\mathrm{e}}$ siècle introduit de fait un point de bascule en ce qu'elle offre au chercheur la possibilité d'entendre le passé. Cette potentialité est pleine de pièges, certes, pour qui oublierait que les sons capturés par les machines ne sont pas le passé mais seulement une composition sonore de ce dernier, néanmoins elle bouleverse la typologie des sources, à tel point qu'il faut s'interroger sur la capacité à écrire une histoire des sons avant que ces nouveautés technologiques ne soient perfectionnées. L'écrasante majorité des sound studies confirme ce doute en se concentrant sur la période la plus récente de l'histoire humaine. De la même manière, l'usage de la notion par M. Schafer et ses épigones s'inscrit avant tout dans le contexte contemporain: l'homme doit ouvrir son sens auditif à son environnement immédiat.

Dès son invention, pourtant, le paysage sonore est inscrit dans la diachronie. Les soundscapes du passé différent de ceux du présent: c'est même l'un des postulats fondamentaux de M. Schafer. Là où les hommes du passé (autrement dit, tous les individus ayant vécu avant la révolution industrielle et ses conséquences acoustiques, une période qui est par ailleurs antérieure à l'invention des technologies sonores) pouvaient écouter en $h i-f i$, c'est-à-dire en distinguant clairement chaque son, les contemporains doivent se contenter d'un environnement lo- $f i$ dans lequel les sons se superposent les uns aux autres ${ }^{28}$. Selon M. Schafer, ce passage du hi-fi au lo-fi est également celui d'une société rurale à une société majoritairement urbaine. Le soundscape est donc inscrit par son inventeur dans un temps orienté, marqué par la détérioration des conditions d'écoute, une péjoration qu'il pense, dans une certaine mesure, inéluctable. Il est impossible de réinventer les sons d'un passé disparu mais les survivants peuvent être préservés et il est urgent d'agir, au moyen du design sonore, pour que le futur soit moins cacophonique ${ }^{29}$. Les tentatives d'enregistrement auxquels le musicologue et ses élèves se livrèrent avaient une finalité de conservation, celle de fixer, avant leur disparition, des paysages sonores menacés par le monde moderne ${ }^{30}$. La technologie, par bien des aspects à l'origine de la perte du paradis $h i-f i$ originel, se trouvait ainsi mise à profit, dans sa dimension

Studies Today: Where Are We Going ?", in J. Damousi et P. Hamilton (dir.), A Cultural History of Sound, Memory and the Senses, New York, Routledge, 2017, p. 7-22.

27 - Sur l'«invention» de la technologie de transduction sonore, voir J. STERnE, Une histoire de la modernité sonore, op. cit., dans lequel il cherche à relativiser l'effet révolutionnaire de l'invention technologique en l'inscrivant dans le temps long du XIX siècle. 28 - R. M. SCHAFER, Le paysage sonore..., op. cit., p. 86-87.

29 - La dernière partie de l'ouvrage, intitulée «Vers un design sonore» (p. 293-369), pose les prolégomènes de cette nouvelle discipline qui passe notamment par la formation de spécialistes ouverts à la musique du monde.

30 - Le programme Five Villages Soundscape, lancé par le World Soundscape Project en 1975, visait à enregistrer, pour les préserver, les paysages sonores de cinq villages européens: Lesconil (France), Skruv (Suède), Bietigheim-Bissingen (Allemagne), Cembra (Italie) et Dollar (Écosse). 
acoustique, pour la sauvegarde de ce qu'elle était en train de détruire ${ }^{31}$. Démarche archivistique, rapport au temps et conscience d'une différenciation chronologique de l'environnement sonore: sans jamais se revendiquer historien, M. Schafer a, le premier, posé les jalons d'une histoire sonore pour que les spécialistes du passé s'emparent de ses sujets et de ses moyens d'étude.

Lorsque A. Corbin publie en 1994 une enquête consacrée aux sons des cloches, il s'inscrit à première vue dans la démarche engagée par M. Schafer ${ }^{32}$. Le sous-titre de son livre sonne comme une revendication claire de la réception de l'ouvrage du théoricien de la musique dans l'approche de l'historien. Le contexte temporel - le siècle de la révolution industrielle - autant que spatial - les campagnes frappées par les mutations économiques et sociales de leur modernisation - dans lequel celui-ci s'inscrit semble adapté à une vision schaférienne assumée. Aussi le lecteur ne manque-t-il pas d'être surpris en constatant l'absence de référence à M. Schafer, bien que A. Corbin ait à l'évidence connaissance de son œuvre ${ }^{33}$. Tout se passe comme si, quinze ans après que la notion a été popularisée, celle-ci était devenue un bien commun, comme si elle s'était détachée de son inventeur et du sens qu'il lui avait donné. Les raisons de cette absence étonnante sont probablement à chercher dans la liberté que l'historien s'accorde dans l'utilisation du «paysage sonore». Du peu de définition que le compositeur avait donné ressortait la dimension unitaire du lieu et du temps: ce que l'on peut entendre en un lieu donné à un moment déterminé. Or l'enquête menée par l'historien français, riche du travail dans les archives de l'ensemble du territoire national sur un siècle entier, le pousse à un usage métaphorique de la notion. Le paysage sonore d'A. Corbin est la reconstruction d'une myriade de prises de son capturées par les documents d'archives, ces ancêtres des disques et des cylindres qui, maniés avec prudence, peuvent tenir lieu d'enregistrements. Ils sont assemblés par l'auteur en un vaste ensemble artificiel, un paysage composé à l'échelle nationale et donc surdimensionnée pour une écoute locale. Par ailleurs, l'enquête porte moins sur le son produit par les cloches, qui n'est évoqué qu'à la marge, que sur les schémas sociaux et culturels que l'objet cloche cristallise. En d'autres termes, A. Corbin est moins intéressé par le son que par le "paysage des activités ${ }^{34}$ » qu'il induit et l'on saisit mieux ainsi sa prise de distance, forcément consciente bien que non explicite, par rapport à M. Schafer. L'historien s'empare donc de la fausse évidence conceptuelle du

31 - Sur le rapport à la mort des technologies de conservation acoustique ainsi que sur la dimension politique de la sauvegarde des sons en voie de disparition, voir J. STERNE, "Une tombe résonnante ", Une histoire de la modernité sonore, op. cit., p. 409-476.

32 - Pour une première réflexion sur les rapports entre les deux auteurs, voir la première partie de l'article de C. VENDRIES, « Du bruit dans la cité... », art. cit., p. 210-214.

33 - Le nom de M. Schafer apparaît régulièrement dans les livres d'entretien que A. CORBIN publia à la suite des Cloches de la terre: Historien du sensible..., op. cit., p. 60-61 (on s'étonne toutefois du qualificatif d'« historien » généreusement accordé au compositeur, voir infra, p. 13) et 116; L'homme dans le paysage..., op. cit., p. 29.

34 - Anthony Pecqueux, «Le son des choses, les bruits de la ville », Communications, 90, 2012, p. 5-16, ici p. 8. Voir, sur ce point, l'analyse historiographique d'Éric PaLAZZo, « Les cinq sens au Moyen Âge. État de la question et perspectives de recherche ", Cahiers de civilisation médiévale, 55-220, 2012, p. 339-366. 
soundscape, tout en se distinguant de l'héritage de son inventeur, ce qui peut se comprendre si l'on se situe strictement sur le plan de la méthode historique. En effet, afin de mettre en avant la dimension diachronique du paysage sonore, M. Schafer adopte, dans les six premiers chapitres de son ouvrage de 1977, une progression chronologique qui le conduit du «paysage sonore naturel » à la «révolution électrique ${ }^{35}$. L'histoire sonore de l'humanité se trouve condensée en quelques dizaines de pages dans lesquelles, au fil des besoins de la démonstration et des connaissances littéraires de l'auteur, Virgile côtoie Robert Stevenson, Bède le Vénérable, Cervantès, et Maxime Gorki, la Bible. L'absence d'une conscience chronologique fine au service d'une démonstration sur le temps long se double, chez M. Schafer, d'une confiance naïve dans les sources littéraires, acceptées comme le miroir non déformant d'un passé révolu.

\section{Silence et émotion}

Le même rapport aux sources se retrouve pourtant au cœur de la démarche de l'Histoire du silence d'A. Corbin, composée elle aussi d'un foisonnement d'extraits d'œuvres littéraires, romans et poésies, dont les citations sont enchaînées avec une grande virtuosité mais peu d'analyse ${ }^{36}$. L'historien paraît ainsi revenir sur une partie des réticences méthodologiques formulées envers le musicologue, qui se dégageaient de la méthode des Cloches de la terre. Si l'on cherche encore en vain le nom de M. Schafer dans l'apparat critique du dernier opus, l'expression «paysage sonore» y est utilisée à plusieurs reprises et la démarche cherchant à "faire ressentir » passe par le déploiement d'un stratagème méthodologique proche de celui mis en œuvre dans Le paysage sonore ${ }^{37}$. Or l'on pourrait adresser à A. Corbin les mêmes reproches qu'à $M$. Schafer concernant la représentativité des passages convoqués. Si, à l'évidence, le nombre de citations mentionnées par l'historien permet d'augmenter la solidité de sa démonstration, leur concentration autour des œuvres de fiction ou des récits intimes censés exalter l'expression des affects induit un biais non négligeable. Confier à Jean des Esseintes, le héros du roman de Joris-Karl Huysmans, la fonction de décrire le silence rêvé d'une chambre à coucher, ou encore à Thérèse d'Avila, celle de dépeindre le silence nécessaire à la contemplation divine, est tout sauf anodin ${ }^{38}$. Puisqu'il est question de «faire ressentir », la parole est donnée aux écrivains, aux poètes ou aux mystiques, pour qui le silence

35 - R. M. SCHAFER, Le paysage sonore..., op. cit., p. 39-150: ces chapitres occupent les parties intitulées «Les premiers paysages sonores » et «Le paysage post-industriel».

36 - A. CORBIN avait pourtant déjà dressé un bilan critique de la méthode adoptée dans Les filles de noces. Misère sexuelle et prostitution, XIX siècle, Paris, Aubier Montaigne, 1978. Dans Historien du sensible..., op. cit., p. 46, il reconnut avoir « commis une erreur » à propos des sources littéraires: «les prendre trop au pied de la lettre et $[\ldots]$ considérer qu'elles avaient implicitement un statut de preuve».

37 - Ibid., p. 56, 93, 96 et 158.

38 - Joris-Karl Huysmans est abondamment utilisé dans A. CorbIn, Histoire du silence..., op. cit., en l'occurrence p. 17-18, citant Joris-Karl HuYsmans, À rebours, Paris, Gallimard, 1983, p. $142-143$; p. 70 pour Thérèse d'Avila. 
est moins un objet en soi que le point de départ de l'évocation des affects qu'il suscite. Ainsi, la méthode utilisée semble adaptée avant tout à l'écriture d'une part bien spéciale de l'histoire des émotions, celle des émotions suscitées par le silence, de la Renaissance à nos jours. Nul doute que la proximité temporelle entre la publication de l'Histoire du silence et celle de l'Histoire des émotions a dû jouer dans la construction du sujet ${ }^{39}$. Mais le silence est-il une émotion ? Défini de manière liminaire comme n'étant "pas seulement une absence de bruit ${ }^{40}$ ", le silence est fondamentalement une catégorie du sonore dont l'étude ne peut pas se résumer à celle des émotions qu'elle suscite.

En donnant abondamment la parole aux contemporains de la période qu'il étudie, A. Corbin permet toutefois de contrecarrer à raison une approche naturaliste des phénomènes sonores. Admirablement résumée par J. Sterne dans les pages liminaires de son Histoire de la modernité sonore, cette tendance conduit à séparer les émissions sonores de leur perception, une séparation totalement impossible pour les sciences sociales ${ }^{41}$. De même que les historiens du visuel n'écrivent pas une histoire de la lumière, qui s'intéresse au sonore ne peut, à proprement parler, écrire une histoire du son indépendante de celle de sa perception. Pour qu'un phénomène acoustique soit défini comme tel dans le monde social, il faut qu'il ait été perçu et identifié ainsi par l'un de ses acteurs. Le son est un produit des sens humains et la seule manière de travailler sur des sons passés est d'asseoir l'enquête sur les perceptions de leurs contemporains ${ }^{42}$. Ainsi, c'est avant tout à une histoire de l'audition que se rattache celle du silence, une histoire infiniment plus complexe que celle d'une réalité sonore que l'on pourrait mesurer, une histoire où la construction sociale des sens a toute sa place, une histoire qui ne saurait se résumer à ce que suscite le silence mais s'intéresse à ce qui permet de le définir dans les perceptions des contemporains.

Pleinement intégrée à l'étude des perceptions, l'histoire du silence se voit donc nécessairement nourrie par les acquis de ces dernières décennies dans les champs de l'histoire et de l'anthropologie des sens. La nécessité d'une appréhension topique des perceptions, aussi bien dans le temps que dans l'espace, a déjà été abondamment démontrée ailleurs. Ainsi, de même que le peuple Massim et celui du cours moyen du Sepik, en Papouasie-Nouvelle-Guinée, diffèrent dans leurs manières de sentir le monde, les perceptions évoluent chronologiquement ${ }^{43}$. La formule de Karl Marx selon laquelle «la formation des cinq sens est le travail de toute l'histoire passée» condense en réalité par anticipation les études d'histoire

39 - Alain Corbin, Jean-Jacques Courtine et Georges Vigarello (dir.), Histoire des émotions, Paris, Le Seuil, 2016. La méthode n'est toutefois pas revendiquée comme telle par les éditeurs.

40 - A. CORBIN, Histoire du silence..., op. cit., p. 9.

41 - J. STERNE, Une histoire de la modernité sonore, op. cit., p. 25-34.

42 - Ibid., p. 21 : «Élément d'un plus vaste phénomène vibratoire physique, le son est un produit des sens humains, non un élément du monde extérieur à l'univers humain. Il ne s'agit que d'une infime partie des vibrations du monde.»

43 - Voir la comparaison proposée par D. Howes, Sensual Relations..., op. cit., p. 160-172. 
des sens ${ }^{44}$. Pour toutes ces raisons, l'objet silence mérite mieux qu'un simple inventaire des «textures ${ }^{45}$ ». Il convient au contraire d'intégrer l'évolutivité de ses définitions locales au cœur même de son étude, à l'image de ce que David Le Breton a pu réaliser pour l'anthropologie ${ }^{46}$. En effet, comment se satisfaire avec A. Corbin d'une chronologie binaire fondée sur un "avant/après » que ne renierait pas M. Schafer, conduisant à la conclusion que «dans le passé les hommes d'Occident goûtaient la profondeur et les saveurs du silence ${ }^{47}$ »?

La nécessité d'une contextualisation fine appelle à un dernier chassé-croisé avec M. Schafer, en lien avec une réévaluation récente de son approche dans la perspective des études historiques. Au terme d'une réflexion sur l'efficacité de la notion de "paysage sonore » et sa pérennité, il est apparu qu'une définition plus précise pourrait avoir comme vertu de la rendre effectivement pertinente pour les sciences sociales ${ }^{48}$. Il a ainsi été proposé, en tenant compte des avatars de son utilisation depuis sa naissance, de comprendre le paysage sonore comme la représentation par un individu ou un groupe d'individus, dont les sens sont le produit d'une construction sociale historiquement datée et contextualisée, d'un ensemble d'événements sonores entendus en un lieu et en un temps déterminés ${ }^{49}$. Une telle caractérisation tient compte des spécificités de l'approche historienne, pour laquelle les sonorités n'apparaissent que par la médiation de sources intrinsèquement limitées dans leur richesse par l'identité de leur producteur. Chaque scène entendue et reproduite dans les sources, y compris écrites, constitue un instantané sonore dont la combinaison permet de reformer la complexité et la profondeur d'un paysage sonore. Toutefois, pour que celui-ci ait une valeur, le cadre d'audition doit être resserré, dans le temps et dans l'espace, et former ce que je propose d'appeler un

44 - Karl Marx, Euvres complètes, vol. 7, Manuscrits de 1844, économie politique et philosophie, Paris, Éd. sociales, 1962, p. 93-94. L'abondance de la bibliographie en histoire et en anthropologie des sens rend illusoire toute idée de synthèse. Outre les différentes publications de D. Howes déjà mentionnées, voir Constance Classen (dir.), A Cultural History of the Senses, Londres, Bloomsbury, 2014, et surtout la page dédiée à l'actualité bibliographique en lien avec la revue The Senses and Society: http://www.sensorystudies. org/books-of-note/. Pour le domaine francophone, voir Ulrike KRAMPL, Robert BECK et Emmanuelle Retaillaud-BajaC (dir.), Les cinq sens de la ville du Moyen Âge à nos jours, Tours, Presses universitaires François-Rabelais, 2013 ; J. CANDAU et M.-B. LE GONIDEC (dir.), Paysages sensoriels..., op. cit.; Marie-Luce GÉLARD, « L'anthropologie sensorielle en France. Un champ en devenir ? ", L'Homme, 216-1, 2016, p. 91-107.

45 - Le terme est omniprésent dans A. Corbin, Histoire du silence..., op. cit., sans que l'analogie ne fasse l'objet d'une véritable explicitation. Il désigne probablement les valeurs assignées au silence et aux divers affects suscités par lui.

46 - David Le Breton, Du silence, Paris, Métailié, [1997] 2015. L'ouvrage convainc avant tout dans sa démonstration des variations civilisationnelles du silence. L'idée d'une évolution dans le temps est moins présente; on s'étonne toutefois, comme pour M. Schafer, de l'absence de référence aux travaux de D. Le Breton dans l'Histoire du silence.

47 - A. Corbin, Histoire du silence..., op. cit., p. 9.

48 - La réflexion collective a été menée dans le cadre du programme de recherche «Paysages sonores et espaces urbains de la Méditerranée antique», commun à l’École française de Rome, l'École française d'Athènes et l'Institut français d'archéologie orien- 
«sonochronotope». Délaissant une grille de lecture purement acoustique au profit de l'interaction entre l'environnement et le sujet, entre le perçu et le percevant, l'historien doit s'autoriser des enquêtes fines sur des cas d'études concentrés sur quelques années, voire quelques décennies, selon la quantité de matériau à disposition. Ainsi peut-on voir s'effacer le risque de construire une «non-historicité des modalités de l'attention, des seuils de la perception, de la signification des bruits, de la configuration du tolérable et de l'intolérable ${ }^{50} \gg$. Encore faut-il pour cela laisser au sujet toutes les possibilités de s'exprimer, autrement dit ne pas le cantonner au rôle de producteur d'affects, mais prendre en considération des situations - et les sources les rapportant - qui ne seraient pas liées à une quête esthétique d'intériorité, dont le silence serait un adjuvant.

\section{Une tentative d'application: le silence rituel de la religion romaine}

Les quelques considérations méthodologiques qui précèdent constituent un appel pour les collègues modernistes et contemporanéistes. Faute d'avoir les capacités de m'en saisir pour les périodes récentes, je propose, à titre de comparaison, certains éléments d'une enquête en cours sur le silence dans l'Antiquité romaine. Bien qu'étant moins fréquenté que celui des autres périodes historiques, le sentier des sensations romaines antiques commence à être régulièrement parcouru ${ }^{51}$. Là, comme ailleurs, les traces abondent pour tenter de réfléchir aux inflexions connues par les modes de perception au cours du temps. Dépassant les a priori d'une faiblesse documentaire, les derniers travaux ont souligné combien les sources sont riches de témoignages permettant des enquêtes sur les perceptions sonores dans l'Antiquité ${ }^{52}$. Au sein de cet ensemble d'attestations qui laisse apparaître des variations dans la signification du silence selon les contextes, celui-ci reste un objet historique à construire dans une approche pluridisciplinaire. Si quelques contributions lui ont déjà été consacrées, particulièrement dans une optique littéraire,

50 - A. CORBIN, «Histoire et anthropologie...», art. cit., p. 230. L'auteur critiquait la partie de l'enquête menée par Guy Thuilliter, Pour une histoire du quotidien au XIX $X^{\ell}$ siècle en Nivernais, Paris, EHEss, 1977, consacrée aux paysages sonores, à laquelle il reprochait un trop grand positivisme.

51 - Eleanor BETTS (dir.), Senses of the Empire: Multisensory Approaches to Roman Culture, Londres, Routledge, 2017; Jerry TONER (dir.), A Cultural History of the Senses in Antiquity, vol. 1, In Antiquity, Londres, Bloomsbury Academic, 2014. L'œuvre collective en cours la plus importante est la collection coordonnée par Mark Bradley, The Senses in Antiquity, Londres/Durham, Routledge/Acumen: Shane Butler et Alex Purves (dir.), Synaesthesia and the Ancient Senses, 2014; Mark BRADLEY (dir.), Smell and the Ancient Senses, 2014; Michael SQuire (dir.), Sight and the Ancient Senses, 2015.

52 - Voir les contributions rassemblées dans la deuxième partie de S. EMERIT, S. PERROT et A. VINCENT (dir.), Le paysage sonore..., op. cit., p. 155-256, plus particulièrement celle de C. VEndries, «Du bruit dans la cité... », art. cit., pour l'Antiquité romaine. 
beaucoup reste à faire pour en comprendre les définitions anciennes, les usages sociaux et les variations contextuelles ${ }^{53}$. L'ambition d'un tel sujet ne peut être contenue dans un article, aussi est-ce à une catégorie de silence particulière que les lignes suivantes sont consacrées, en dialogue avec le parti pris d'A. Corbin ${ }^{54}$. Pour ce dernier, le silence est de manière évidente un élément clé de la vie intérieure et un mode d'accès au divin ${ }^{55}$. Une telle énonciation, dans sa dimension essentialiste, ne manque pas de questionner sur la longue durée et il n'est donc pas illégitime de porter l'interrogation sur des temps éloignés, voire antérieurs au développement du rapport chrétien au silence ${ }^{56}$. Quel(s) rôle(s) les Romains assignaient-ils

53 - Sans prétendre à l'exhaustivité, voir notamment Concepción BERMEJO JIMÉNEZ, «El silencio en Tibulo », in Simposio Tibuliano. Commemoración del bimilenario de la muerte de Tibulo, Murcie, Universidad de Murcia, 1985, p. 217-225 ; Marie LeDENTU, « Horace et la vertu du silence, des Satires aux Odes romaines. Questionnements éthiques et esthétiques sur les enjeux de la libertas et de l'amicitia dans l'entourage d'Octavien/Auguste ", Latomus. Revue d'études latines, 73-2, 2014, p. 399-414; Roberta STrocchio, I significati del silenzio nell'opera di Tacito, Turin, Accademia delle scienze, 1992; Valentina CHINNICI, «La dialettica fra suono e silenzio in Calpurnio Siculo», in L. LANDOLFI et R. ODdo (dir.), Fer propius tua lumina. Giochi intertestuali nella poesia di Calpurnio Siculo, Bologne, Pàtron, 2009, p. 129-142; Géraldine Puccini-Delbey, "La vertu de silence dans les Métamorphoses d'Apulée", in R. Poignault (dir.), Présence du roman grec et latin, Clermont-Ferrand, Centre de recherches A. Piganiol-Présence de l'Antiquité, 2011, p. 225-236; Yoneko NurTantio, Le silence dans l'Énéide, Bruxelles, Éd. modulaires européennes, 2014; Jacques Heurgon, «Le silence tragique de Didon (Énéide VI, 450-476) », in Mélanges de philosophie, de littérature et d'histoire ancienne offerts à Pierre Boyancé, Rome, École française de Rome, 1974, p. 395-400; Otto SEEL, Quintilian oder die Kunst des Redens und Schweigens, Stuttgart, Klett-Cotta, 1977; Thomas Köves-Zulauf, «Reden und Schweigen im taciteischen Dialogus de oratoribus», Rheinisches Museum für Philologie, 135, 1992, p. 316-341; Christina Shuttleworth KRAUS, « Reden und Schweigen in Caesars Bellum Gallicum », in T. Fuhrer et D. Nelis (dir.), Acting with Words: Communication, Rhetorical Performance and Performative Acts in Latin Literature, Heidelberg, Universitätsverlag Winter, 2010, p. 9-30; Robert J. BAKER, «Sallustian Silence », Latomus. Revue d'études latines, 41-4, 1982, p. 801-802; Gernot KraPInGER, "Schweigen in den Viten Plutarchs", in S. JÄKEL et A. TimONEN (dir.), The Language of Silence, Turku, Turun Yliopisto, 2001, vol. 1, p. 105-112; Benjamin E. STEVENS, Silence in Catullus, Madison, University of Wisconsin, 2013; Elżbieta WesoŁowsKa, "Silence in Seneca's Tragedies », Acta antiqua Academiae Scientiarum Hungaricae, 33, 1990-1992, p. 77 81; Ernest DutorT, «Silences, dans l'œuvre de Tite Live », in Mélanges de philologie, de littérature et d'histoire anciennes, offerts à J. Marouzau par ses collègues et élèves étrangers, Paris, Les Belles Lettres, 1948, p. 141-151; Yelena BARAZ, "Sound and Silence in Calpurnius Siculus ", American Journal of Philology, 136, 2015, p. 91-120; Pasqualina VozzA, «Un silenzio eloquente (quid tacitus...? Calp. Ecl. 4, 1-4)», Bollettino di studi latini, 24-1, 1994, p. 71-92; Ewa Skwara, «What Does Silence Say? Functions of Silence in Plautus' Comedies », Eos. Commentarii Societatis Philologae Polonorum, 87-2, 2000, p. 279-286. 54 - Une enquête plus globale sur le silence dans l'Antiquité romaine est en cours.

55 - Pour A. Corbin, Histoire du silence..., op. cit., p. 30, l'évidence est telle qu'elle n'appelle même pas le commentaire: «Passons rapidement sur ce qui unit le silence à la liturgie, tant cela va de soi. »

56 - On attend avec impatience la publication imminente de l'ouvrage que Vincent Debiais consacrera à cette question. 
au silence dans les rites de leur religion publique ${ }^{57}$ ? Pour répondre à cette question, l'historien de l'Antiquité doit faire feu de tout bois et utiliser tous les types de sources à sa disposition : correspondances, traités, narrations historiques, commentaires, documents iconographiques, etc. La période qui est davantage documentée correspond au dernier siècle de la République et au premier du Principat $\left(\mathrm{I}^{\mathrm{er}}\right.$ siècle av. J.-C.-- ${ }^{\text {er }}$ siècle apr. J.-C.. ${ }^{58}$.

La langue latine dispose de deux séries de termes pour désigner la catégorie sonore que le français traduit par silence: le verbe silere et ses dérivés, dont le substantif silentium; le verbe tacere et sa famille, comprenant le substantif taciturnitas. L'équivalence entre les deux champs n'est pas exacte et une étude lexicographique serrée reste à mener ${ }^{59}$, mais la définition la plus simple est celle de l'absence de son, ainsi que l'écrit Isidore de Séville dans ses Étymologies ${ }^{60}$. Sans grande surprise, les sources localisent donc avant tout le silence à la campagne. C'est là, de préférence dans les bois, que se réfugie Pline le Jeune lorsqu'il souhaite retrouver le calme de l'inspiration ${ }^{61}$; là encore que fuit le chœur des poètes conduit par Horace dès lors qu'il est question de déserter la ville qu'agite un "vacarme de jour comme de nuit ${ }^{62}{ }$. La Rome du I $^{\text {er }}$ siècle apr. J.-C., peuplée d'environ un million d'habitants, est présentée dans les sources comme un maelström sonore où le repos est difficile à trouver, du moins pour qui ne possède pas les ressources d'une domus isolée ${ }^{63}$. Si les bruits des activités de production sont visés par les

57 - Les rapports entre religion romaine et silence n'ont, à ce jour, pas fait l'objet d'un traitement en soi. Il faut noter la publication d'un séminaire d'histoire des religions: Santiago Montero et María Cruz Cardete (dir.), Religión y silencio. El silencio en las religiones antiguas, Madrid, Universidad Complutense de Madrid, 2007. La présentation bibliographique liminaire faite par Marcos Rodriguez Plaza (p. 16-18 pour l'Antiquité romaine) est éloquente quant au travail qui reste à accomplir. Voir aussi Michel Humm, "Silences et bruits autour de la prise d'auspices », in M.-T. SchetTino et S. PitTia (dir.), Les sons du pouvoir dans les mondes anciens, Besançon, Presses universitaires de FrancheComté, 2012, p. 275-295.

58 - On ne s'interdira évidemment pas l'utilisation de commentaires postérieurs, comme ceux du scoliaste Servius sur l'Énéide, dans la mesure où ils permettent un éclaircissement pertinent. De même trouve-t-on des éléments chez Festus, un auteur tardif qui reprend les œuvres de l'auteur augustéen Marcus Verrius Flaccus.

59 - Les bases en ont été posées par Arduino MaIURI, «La polisemia del silenzio nel mondo latino tra politica, diritto e religione ", in Silenzio e parola nella patristica, Rome, Institutum Patristicum Augustinianum, 2012, p. 465-486.

60 - ISIDORE DE SÉVILLE, Étymologies, 13, 10, 12: silentium non aliqua res est, sed uti sonus non est, silentium dicitur.

61 - Pline Le Jeune, Lettres, 1, 6, 2.

62 - Horace, Épitres, 2, 2, 77-80: Scriptorum chorus omnis amat nemus et fugit urbem rite cliens Bacchi somno gaudentis et umbra, tu me inter strepitus nocturnos atque diurnos uis canere et contracta sequi uestigia uatum?

63 - Martial, Épigrammes, 12, 57, 3-4 ; Juvénal, Satires, 3, 234-235. Les sources satiriques sont les premières concernées. Si leur nature suppose l'exagération, elles reposent malgré tout sur un fondement acceptable. Voir leur utilisation par Antonio GONZALES, "Discours contre le bruit, discours contre l'autre dans une mégalopole antique: le cas de Rome », in I. Pimouguet-Pédarros, M. Clavel-Lévêque et F. Ouachour (dir.), 
attaques des satiristes ${ }^{64}$, c'est avant tout la voix humaine qui est l'ennemie du silence en ville, notamment à travers les cris des haleurs le long du Tibre ${ }^{65}$, ceux des marchands d'animaux en route pour les marchés ${ }^{66}$, ou encore ceux des bateliers se disputant le chaland ${ }^{67}$. La Lettre 56 de Sénèque, dans laquelle le philosophe démontre la capacité du sage stoïcien à réfléchir dans des conditions acoustiques extrêmes, est, à ce titre, la meilleure anticipation d'une littérature postérieure faisant connaître la ville par ses cris : marchands, épilateurs, baigneurs jouent sans cesse de la voix ${ }^{68}$. L'opposition entre le silence et la voix dépasse le cadre urbain. C'est dans l'absence d'émission vocale, en réalité, que semble résider la véritable définition du silentium et de la taciturnitas antiques ${ }^{69}$. Le silence est ainsi l'opposé du discours, qu'il soit pensé comme tel par l'orateur qui l'utilise comme une arme rhétorique ${ }^{70}$, imposé à la foule par le héraut avant qu'un magistrat ne prenne la parole, ou suscité par une émotion si forte qu'elle laisse un individu sans voix, voire une foule entière dans certaines circonstances exceptionnelles : c'est le « silence des grandes peurs et des grandes colères » qu'observe, par exemple, la plèbe tacitéenne rassemblée sur le forum pour assister aux pathétiques derniers instants de l'éphémère empereur Galba ${ }^{71}$.

La place du silence dans les rites de la religion romaine peut être énoncée de manière simple en suivant la glose à l'Énéide rédigée au IV siècle par Servius: «en effet le silence était nécessaire aux cérémonies religieuses ${ }^{72}{ }$. L'affirmation masque toutefois une diversité de situations que révèle l'objet même du

Hommes, cultures et paysages de l'Antiquité à la période moderne. Mélanges offerts à Jean Peyras, Rennes, PuR, 2012, p. 243-258.

64 - Martial, Épigrammes, 12, 57, 5-6, se plaint des bruits nocturnes de la boulangerie mais surtout des activités touchant au métal: la chaudronnerie (voir aussi 9, 69, la fabrication de statue de bronze) ou le change de monnaie. SÉNÈQue, Lettres à Lucilius, 56,4 , mentionne un serrurier. Dans le même texte, il fait état d'un facteur essayant ses instruments de musique.

65 - MARTIAL, Épigrammes, 4, 64, 21-22.

66 - JuvénAL, Satires, 3, 236-238.

67 - Horace, Satires, 15, 11-13.

68 - Pour une analyse de ce célèbre document, le plus abondamment cité sur la question des bruits urbains, qu'on me permette de renvoyer en dernier lieu à Alexandre Vincent, «Les silences de Sénèque », Pallas. Revue d'études antiques, 98, 2015, p. 131143. Le plus fameux exemple d'appréhension d'une ville par les sons de ses habitants se trouve probablement dans le Tableau de Paris de Louis-Sébastien Mercier. Un très bel exemple médiéval, le Concile d'Apostoile ou Grand riote (BNF, ms. fr. 837 et 19152), a été récemment étudié par Jean-Marie FriTZ, «Paysage sonore et littérature médiévale: fécondité et fragilité d'une rencontre ", in L. HABLOT et L. VISSIÈRE (dir.), Les paysages sonores. Du Moyen Âge à la Renaissance, Rennes, PuR, 2015, p. 299-301.

69 - Pour le monde grec, voir Silvia MonTiglio, Silence in the Land of Logos, Princeton, Princeton University Press, 2010.

70 - Henri BARDON, «Le silence, moyen d'expression ", Revue des études latines, 21-22, 1943-1944, p. 112-120.

71 - TACITE, Histoires, 1, 40, 1.

72 - Servius, In Verg., 5, 71, Teubner, Leipzig, 1881 (trad. de l'auteur) : nam in sacris 
commentaire du grammairien, qui porte sur un vers dans lequel Virgile place dans la bouche d'Énée les mots ore fauete ${ }^{73}$. Cette expression, que l'on traduirait littéralement par «que votre bouche soit favorable», est rare: seul Ovide l'utilise quelques années après le poète lombard ${ }^{74}$. La même idée se rencontre plus fréquemment sous la forme fauete linguis, qui posa tout autant problème aux Anciens eux-mêmes ${ }^{75}$. Sénèque, dans un passage de son traité $D e$ la vie heureuse, fournit un précédent à Servius: il faut que le silence se fasse afin que les rites puissent être réalisés correctement, c'est-à-dire sans qu'ils ne soient perturbés par des paroles inadaptées. Le philosophe recourt à une étymologie étonnante qui revient à considérer fauete linguis comme le synonyme exact de "faire le silence »: «Cette expression ne vient pas, comme on le croit généralement, de 'faveur', mais elle commande le silence (imperat silentium) pour que la cérémonie religieuse puisse être accomplie selon le rite, sans qu'aucun mot fâcheux ne la trouble ${ }^{76}$.» En semblant nier que fauere soit un parent de fauor, Sénèque adopte une position linguistique délicate et radicale, que ne reprend pas l'abréviateur de Festus, Paul Diacre, selon qui «fauere signifie dire des paroles favorables, mais les anciens poètes ont utilisé fauere pour silere ${ }^{77}$ ». Servius, dans la suite du passage mentionné, occupe une position intermédiaire en faisant état des deux possibilités d'interprétation: " 'fauete linguis, fauete uocibus', prononcez des paroles de bon présage ou taisezvous $^{78}$ ». Les alternatives d'interprétation peuvent étonner : s'agit-il de parler (pour prononcer des paroles adaptées, considérées comme favorables) ou de se taire? En réalité, l'idée reste la même et repose sur l'efficacité de la parole. Il s'agit, par l'énonciation de mots idoines ou la rétention de paroles omineuses, de créer autour du rite un environnement sonore propice à sa réalisation, selon les prescriptions du droit religieux. Une compréhension plus profonde de ce qu'était le silence religieux nécessite toutefois de regarder avec précision le contexte dans lequel ce dernier apparaît dans les sources: pour quels rites en est-il fait état et à quel moment du rite?

73 - Virgile clôt ainsi le discours d'Énée instituant des jeux funéraires pour son père Anchise.

74 - OvIDE, Ibis, 98-99: Quisquis ades sacris, ore fauete, meis ; quisquis ades sacris, lugubria dicite uerba. La construction similaire des deux vers est évidemment frappante.

75 - Francesca Prescendi, Décrire et comprendre le sacrifice. Les réflexions des Romains sur leur propre religion à partir de la littérature antiquaire, Stuttgart, Franz Steiner, 2007, p. $72-73$.

76 - SÉNÈQue, De la vie heureuse, 26, 7, 8 (trad. modifiée par l'auteur): Hoc uerbum non, ut plerique existimant, a fauore trahitur, sed imperat silentium ut rite peragi possit sacrum nulla uoce mala obstrepente.

77 - PAul Diacre, in Festus, 78 L. (trad. de l'auteur): Fauere enim bona fari, at ueteres poetae pro silere usi sunt fauere. Bien que l'expression n'y apparaisse pas, il faut aussi penser au fauete linguis dans un autre lemme de Paul Diacre, in Festus, 249 L.: Parcito linguam in sacrificiis dicebatur, id est coerceto, contineto, taceto.

78 - Servius, in Verg., 5, 71 (trad. de l'auteur) : «fauete linguis, fauete uocibus », hoc est bona omina habete aut tacete. L'expression fauete uocibus est un hapax, qui ne semble avoir d'autre vertu que d'expliciter fauete linguis en élargissant la métonymie. 
Les éléments présentés, qui lient silentium et sacrum, désignent les cérémonies religieuses en général, suggérant que tous les rites de la religion publique nécessitaient au moins un temps de silence. C'est aussi ce que laisse penser Cicéron dans un passage de son traité De la divination, dans lequel il rappelle les bons préceptes des Anciens:

nos ancêtres, considérant que ces présages ont de la valeur, faisaient précéder toutes leurs actions de la formule : "Que cela soit bon, favorable, heureux et suivi de succès »; pour des cérémonies religieuses publiques, on commandait: «Faites silence!»(fauerent linguis) et, dans l'annonce des fêtes, "qu'on s'abstînt de procès et de querelles " ${ }^{79}$.

L'expression utilisée par Cicéron pour désigner le contexte d'apparition du silence, rebus diuinis quae publice fierent, est encore plus générale que sacrum. Une lecture attentive des sources permet de constater qu'en réalité, dans leur quasi-totalité, celles-ci associent le silence à deux types de rite particuliers: la prise d'auspices et la réalisation d'un sacrifice. Un passage de Plutarque s'en fait l'écho:

Les Pythagoriciens ne permettaient pas, dit-on, d'adorer et de prier les dieux pour ainsi dire en passant; ils voulaient qu'on s'y préparât en y pensant dès que l'on sortait de chez soi. Numa estimait de même que les citoyens ne doivent entendre ni regarder aucune cérémonie sacrée à la légère et avec négligence, mais quitter toute autre occupation et appliquer leur esprit à la piété, comme étant l'affaire de toutes la plus importante, suspendre, enfin, le tumulte, le tapage, les gémissements et tous les bruits du même genre qui accompagnent les travaux manuels indispensables, de façon à en purifier les rues pendant l'accompagnement des rites. Il reste jusqu'à nos jours une trace de cet usage: quand un magistrat s'occupe d'augures ou de sacrifices, on crie "Hoc age» (mots qui signifient «veille à ce que tu fais »), pour rendre les assistants attentifs et recueillis ${ }^{80}$.

Bien que le texte du polygraphe commette une confusion dans la formule prononcée par le héraut - l'expression hoc age était bien énoncée elle aussi mais à un autre

79 - CicÉron, De la divination, 44, 102: Quae maiores nostri quia ualere censebant, idcirco omnibus rebus agendis "quod bonum, faustum, felix fortunatumque esset » praefabantur, rebusque diuinis, quae publice fierent, ut "fauerent linguis", imperabatur inque feriis imperandis, ut "litigibus et iurgiis se abstinerent ».

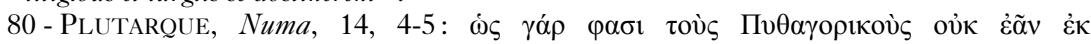

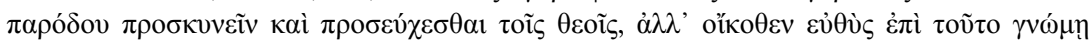

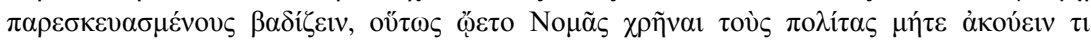

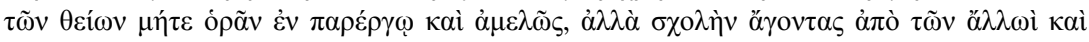

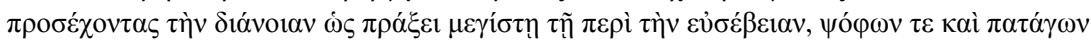

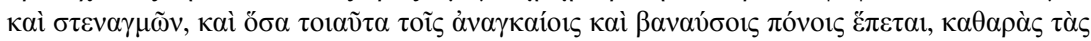

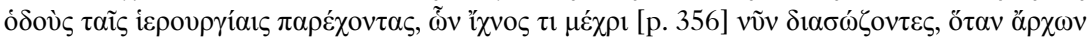

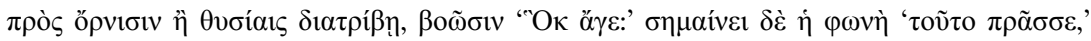

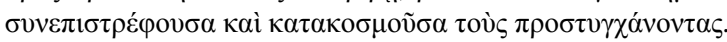


moment du rite et elle ne possédait pas la même fonction que fauete linguis ${ }^{81}$-, il marque avec force la distinction entre deux temporalités de la vie collective, l'une sacrée et l'autre profane, auxquelles correspondraient deux types d'ambiance sonore distinctes, une bruyante et une silencieuse. Mais il faut aller encore plus loin et s'intéresser à la consultation des oiseaux et aux sacrifices, qui ne mettaient pas en œuvre le même silentium.

\section{La perfection du silence augural}

Le premier rite dont Plutarque fit état, la prise des auspices, était un rite divinatoire. Il s'agissait de solliciter les dieux, plus particulièrement Jupiter, afin d'obtenir les signes de leur assentiment quant aux décisions importantes de la cité ${ }^{82}$. La prise des auspices était une technique rituelle (disciplina) grâce à laquelle un magistrat disposant du droit approprié (auspicium) créait les conditions de l'agrément des divinités ${ }^{83}$. Il lui revenait de constater ces conditions par l'observation de la foudre et des éclairs mais surtout par celle du vol des oiseaux et de l'appétit des poulets sacrés. Le magistrat était assisté d'un prêtre dont c'était la fonction, l'augur, puis, plus fréquemment à la fin de la période républicaine, d'un appariteur responsable des poulets sacrés (pullarius) ${ }^{84}$.

Au cours de la prise d'auspices, le silentium se voyait affecté une triple valeur: temporelle, acoustique et juridique. La documentation concernant les auspices suggère qu'ils étaient pris au tout début du jour civil, soit, en réalité, en pleine obscurité, lors d'une période de la nuit qui aurait porté le nom de silentium noctis. Il s'agirait du troisième quart de la nuit, entre ce que les sources désignent comme le concubium et le gallicinum, selon une récente hypothèse de Michel Humm ${ }^{85}$. Ainsi défini comme une unité temporelle nocturne, le silentium n'en était pas moins une véritable catégorie sonore. Il était l'environnement acoustique propice à la réception de signes divins. Le silence a pris un sens particulier dans l'observation d'un type d'auspices désigné, selon le grammairien Festus, par les termes tripudium

81 - F. PRESCENDI, Décrire et comprendre le sacrifice..., op. cit., particulièrement p. 75-76. 82 - La prise des auspices est un temps particulièrement important de la vie collective (religieuse et politique) romaine, qui a suscité une abondante littérature. Voir, en dernier lieu, Yann Berthelet, Gouverner avec les dieux. Autorité, auspices et pouvoir, sous la République romaine et sous Auguste, Paris, Les Belles Lettres, 2015. Sur la question particulière du silence augural, voir M. HuMM, "Silences et bruits..." , art. cit., ainsi que Santiago Montero, «Del silencio augural al silencio ante el prodigio », in $\mathrm{S}$. Montero et M. C. Cardete, Religión y silencio..., op. cit., p. 165-174.

83 - John SCHEID, «La parole des dieux. L'originalité du dialogue des Romains avec leurs dieux », Opus, 6-8, 1987-1989, p. 125-136.

84 - Sur la chronologie et les raisons de cette évolution, voir Y. BERTHELET, Gouverner avec les dieux..., op. cit., p. 220-235. En attendant une véritable enquête sur les pullarii, voir Giuseppina FOTI, «Funzioni e caratteri del 'Pullarius' in età repubblicana e imperiale ", Acme, 64-2, 2011, p. 89-121.

85 - M. HuMM, «Silences et bruits...», art. cit., en particulier p. 288 sur les sources situant la prise des auspices la nuit. 
soninium $^{86}$. Les dieux n'auraient pas manifesté leur volonté par un signe visible, comme c'était le cas pour la majorité d'entre eux, mais sonore : la vacuité acoustique environnante l'aurait rendu plus perceptible ${ }^{87}$. La nature délicate de cette manifestation a permis de mieux comprendre la nécessité d'un silence parfait pour sa réception : il s'agirait, si l'on en croit Cicéron, augure depuis l'année 53-52 av. J.-C., du son émis par les miettes tombant du bec des poulets sacrés lorsqu'elles heurtaient le sol ${ }^{88}$. Le silentium, à la fois unité temporelle et réalité acoustique, marquait la volonté de distinction entre le temps des activités profanes et celui des activités religieuses, que Plutarque fait remonter à Numa, figure royale construite comme celle de l'organisateur de la religion publique romaine, mais qui avait davantage encore de sens aux époques postérieures. Prendre les auspices dans le troisième quart de la nuit, avant que ne résonne le chant du coq et que ne se lève le jour, était la garantie de procéder aux opérations dans le calme d'une cité endormie. Pour les auspices pris à Rome même, il s'agissait d'éviter le brouhaha de la ville, qui devait monter jusqu'à l'auguraculum d'où ils étaient observés - on pense particulièrement à celui situé sur l'Arx, qui surplombait le forum et ses multiples activités ${ }^{89}$.

Toutefois, le silentium était décrit dans les sources non seulement comme le cadre pragmatique de déroulement des rites, mais aussi et surtout comme

86 - Le tripudium soniuium était manifestement explicité dans le traité de droit augural d'Appius Pulcher (mention dans Festus, $382 \mathrm{~L}$ : «Soni> uium tripu<dium [...] Appius $>$ Pulcher, quod [...]»). L'identification exacte des sons qu'il aurait fallu guetter est soumise à discussion. Si l'on suit Servius, In Verg., 3, 90, il s'agissait du bruit produit par la chute d'un rocher ou d'un arbre. PLINE L'ANCIEN, Histoire naturelle, 15, 24 fait état du bruit des noix heurtant le sol.

87 - On trouve un parallèle intéressant dans le célébrissime document des Tables Eugubines, qui affirme la nécessité du silence dans les prises d'auspices de la cité ombrienne. La Table VI précise ainsi : «Quand sur le siège sera assis celui qui ira observer les [oiseaux] criants, dès lors qu'on ne fasse pas de bruit, qu'un tiers ne s'immisce pas jusqu'à ce que soit revenu celui qui sera allé observer les [oiseaux] criants. Si l'on fait du bruit ou [si] quelqu'un d'autre s'est immiscé, [le rituel] sera invalide.» Je reprends ici la traduction et la belle analyse de Jean-Claude LACAM, «L'univers sonore dans les Tables Eugubines », Res Antiquae, 12, 2015, p. 103-116.

88 - CICÉRON, Lettres aux familiers, 6, 6, 7: «Ainsi, puisque, à la manière des augures et des astrologues, j'ai comme eux réussi, moi qui suis augure officiel, à établir à tes yeux, par mes prédictions antérieures, l'autorité de mon art augural et divinatoire, une prédiction de ma façon devra t'inspirer confiance. Ce n'est donc pas de la venue d'un oiseau au vol prophétique, ni du chant 'à gauche' d'un oiseau au cri prophétique, comme cela se pratique dans notre science augurale, ni des miettes qui tombent intactes ou avec bruit du bec des poulets que je tire un présage pour ton avenir; j'ai d'autres signes qui s'offrent à mon observation et, s'ils ne sont pas plus sûrs que les premiers, ils contiennent moins d'obscurité et prêtent moins à méprise.» Il est préférable de suivre Cicéron quant à la nature de cette irruption sonore de l'expression divine plutôt que SERviUs, In Verg., 3, 90, selon qui il s'agissait du bruit produit par la chute d'un rocher ou d'un arbre, assurément plus audible même sans silence environnant.

89 - Les divers anguracula de la ville étaient situés en hauteur, autant pour des raisons religieuses que pratiques, afin de garantir une meilleure observation du vol des oiseaux. Voir la carte de Filippo Coarelli reproduite dans M. Humm, «Silences et bruits... ", art. cit., p. 279. 
une catégorie rituelle nécessaire à son accomplissement. Festus l'exprimait en toute clarté: «car le silentium, c'est l'absence de tout vice de procédure (uitium) dans les auspices ${ }^{90}$ ". Le silentium aurait été une "condition fondamentale», pour reprendre les mots de Georges Dumézil, voire une condition nécessaire à la réalisation parfaite du rite et, donc, à son accomplissement ${ }^{91}$. Cette dimension de perfection rituelle a été transmise de la manière la plus complète par Cicéron, dans sa célèbre description d'une prise d'auspices:

"Quintus Fabius, je veux que tu m'assistes dans la prise d'auspices. "Réponse: "J'ai entendu.»Du temps de nos ancêtres cet assistant était un expert, aujourd'hui c'est n'importe qui. En effet, il faut être un expert pour savoir ce qu'est le silentium (dans les auspices on appelle ainsi l'absence de tout vice). Pour constater le silentium, il faut être un augure achevé. Or quand l'auspicant enjoint à celui qui l'assiste dans la prise d'auspices : « Si le silentium te parầt établi, annonce-le!, celui-ci ne regarde ni vers le haut, ni tout autour; mais répond sur-le-champ que le silentium paraît être réalisé. L'auspicant dit: "S'ils mangent, annonce-le!» - «Ils mangent.»"

La narration de cette prise d'auspices par les poulets sacrés (auspicium ex tripudiis) a été beaucoup commentée, notamment parce qu'elle révélait des signes néfastes, qui n'existent pas tant qu'ils ne sont pas perçus ${ }^{93}$. Les sources permettent d'en dresser la liste suivante: la chute d'un objet - particulièrement la verge d'un licteur ${ }^{94}$-, d'un arbre ou d'un rocher ${ }^{95}$, la perception de cris de souris ${ }^{96}$, le fait qu'un des participants ait eu des vents durant la prise d'auspices ${ }^{97}$, ou que le vent souffle trop fort ${ }^{98}$. Tous ces phénomènes ont une dimension sonore, même le dernier: les augures pouvaient constater par la vue la présence de vent - il ne fallait pas que leur

90 - FesTUS, 474 L.: hoc enim est <proprie sil>entium, omnis uitii in auspiciis uacuitas.

91 - Georges DumÉzIL, La religion romaine archä̈que, Paris, Payot, 1966, p. 589. L'auteur traite des auspices pris par le paterfamilias en contexte privé, ce qui ne change rien à la valeur de l'analyse.

92 - CICÉRON, De la divination, 2, 71-72: «Q. Fabi, te mihi in auspicio esse uolo». Respondet: «Audiui». Hic apud maiores adhibebatur peritus, nunc quilubet. Peritum autem esse necesse est eum qui, silentium quid sit, intellegat; id enim silentium dicimus in auspiciis, quod omni uitio caret. Hoc intellegere perfecti auguris est; illi autem qui in auspicium adhibetur, cum ita imperauit is, qui auspicatur: "Dicito, silentium esse uidebitur", nec suspicit nec circumspicit; statim respondet silentium esse uideri. Tum ille: «Dicito, si pascentur » - «Pascuntur».

93 - F. Prescend, Décrire et comprendre le sacrifice..., op. cit., p. 74 sq., ainsi que S. MONTERO, «Del silencio augural... », art. cit.

94 - Paul Diacre, Festus, 56 L.

95 - Festus, 382 et 386 L.

96 - Pline L'Ancien, Histoire naturelle, 8, 62, 223: Nam soricum occentu dirimi auspicia Annales refertos habemus. Qu'entendre des cris de souris ait été considéré comme un mauvais présage est confirmé par VALÈre MaXime, Faits et dits mémorables, 1, 1, 5, 3: occentusque soricis auditus Fabio Maximo dictaturam, C. Flaminio magisterium equitum deponendi causam praebuit. Le rapport de ces animaux avec le monde souterrain est assez clair pour que l'on comprenne aisément les raisons de ces présages négatifs.

97 - CATON, De l'orateur, fr. 73 (Malcovati) $=60$ (Cugusi).

98 - Plutarque, Questions romaines, 73. 
lampe, toujours découverte, ne vienne à s'éteindre -, mais la puissance du souffle relève aussi du sonore ${ }^{99}$. La perfection du silentium augural, c'est-à-dire l'absence de tout vice, ne pouvait donc se déterminer que par l'audition. Le silentium augural était un silence maximal, si ce n'est absolu. L'augure ou l'appariteur, afin de s'assurer des bonnes conditions d'observation des signa, devait avant tout garder ses oreilles en éveil. Dans ce cas, comment comprendre les vitupérations de Cicéron sur l'incapacité des desservants de son temps? Était-il difficile d'être un «augure achevé », pour reprendre l'Arpinate, s'il ne s'agissait que d'ouvrir ses oreilles? Un simple appariteur comme celui que Cicéron a mis à la manœuvre dans son texte n'était-il pas en mesure de rester à l'aguet du moindre bruit ${ }^{100}$ ? La peritia résidait peut-être moins dans la perception des bruits eux-mêmes que dans la distinction à opérer entre les bruits anodins (une feuille qui tombe, un chien qui aboie au loin...) et les bruits néfastes, selon les prescriptions rituelles. L'expertise requise n'était pas sensorielle mais bien rituelle.

\section{Le silence bruyant des sacrifices}

Le deuxième temps de la vie religieuse pour lequel Plutarque exprimait la nécessité du silence, le sacrifice, a engendré une quantité de sources plus importantes que la prise d'auspices. Cette disproportion se comprend assez aisément en tenant compte du contexte qui appelait les deux rites: les Romains réalisaient des sacrifices bien plus régulièrement qu'ils ne prenaient des auspices. Si les sources ne confèrent pas au silentium des sacrifices une dimension temporelle, elles attestent pour autant du fait qu'il devait être établi pour que le rite soit réalisé justement. Les modalités de création du silentium sacrificiel étaient plus élaborées que pour la prise d'auspices: elles nécessitaient la mise en place de plusieurs stratégies et conduisaient à la création de trois cercles de silence.

Un relief votif actuellement conservé au musée archéologique de Milan en fournit une représentation (fig. 1$)^{101}$. Réalisé par Publius Qurtius Victor et Publius

99 - Columelle, De l'agriculture, 4, 29, 5; 12, 25, 4.

100 - Françoise Van HAEPEREN, «Auspices d'investiture, loi curiate et légitimité des magistrats romains », Cahiers du Centre Gustave-Glotz, 23, 2012, p. 71-111, ici p. 76-77, suppose qu'il devait s'agir des pullarii. C'est aussi le cas de G. FOTI, «Funzioni e caratteri...", art. cit.

101 - Le relief provient du territoire de la commune de Varese (l'antique Angera), mais l'original est conservé au Civico museo archeologico di Milano, n. inv. A6774. La publication la plus récente est Floriana Cantarelli, Catalogo del lapidario dei musei civici di Varese, Musei civici di villa Mirabello, Varese, 1996, $\mathrm{n}^{\circ} 17$ et appendice 2, p. 172-176, qui propose comme datation le deuxième quart du I ${ }^{\text {er }}$ siècle apr. J.-C. Je remercie Anna Provenzali, conservatrice au musée archéologique de Milan, de m'avoir fourni diligemment toutes les informations à sa disposition concernant ce relief, ainsi que la photographie reproduite. De manière générale, pour les représentations du sacrifice romain, voir les travaux de Valérie HuET, plus particulièrement «La mise à mort sacrificielle sur les reliefs romains: une image banalisée et rituelle de la violence? », in J.-M. MARTIN (dir.), La violence dans les mondes grecs et romains, Paris, Publications de la Sorbonne, 2005, p. 91-119. 
Figure 1 - Représentation d'un sacrifice sur un monument votif en marbre dédié à Jupiter

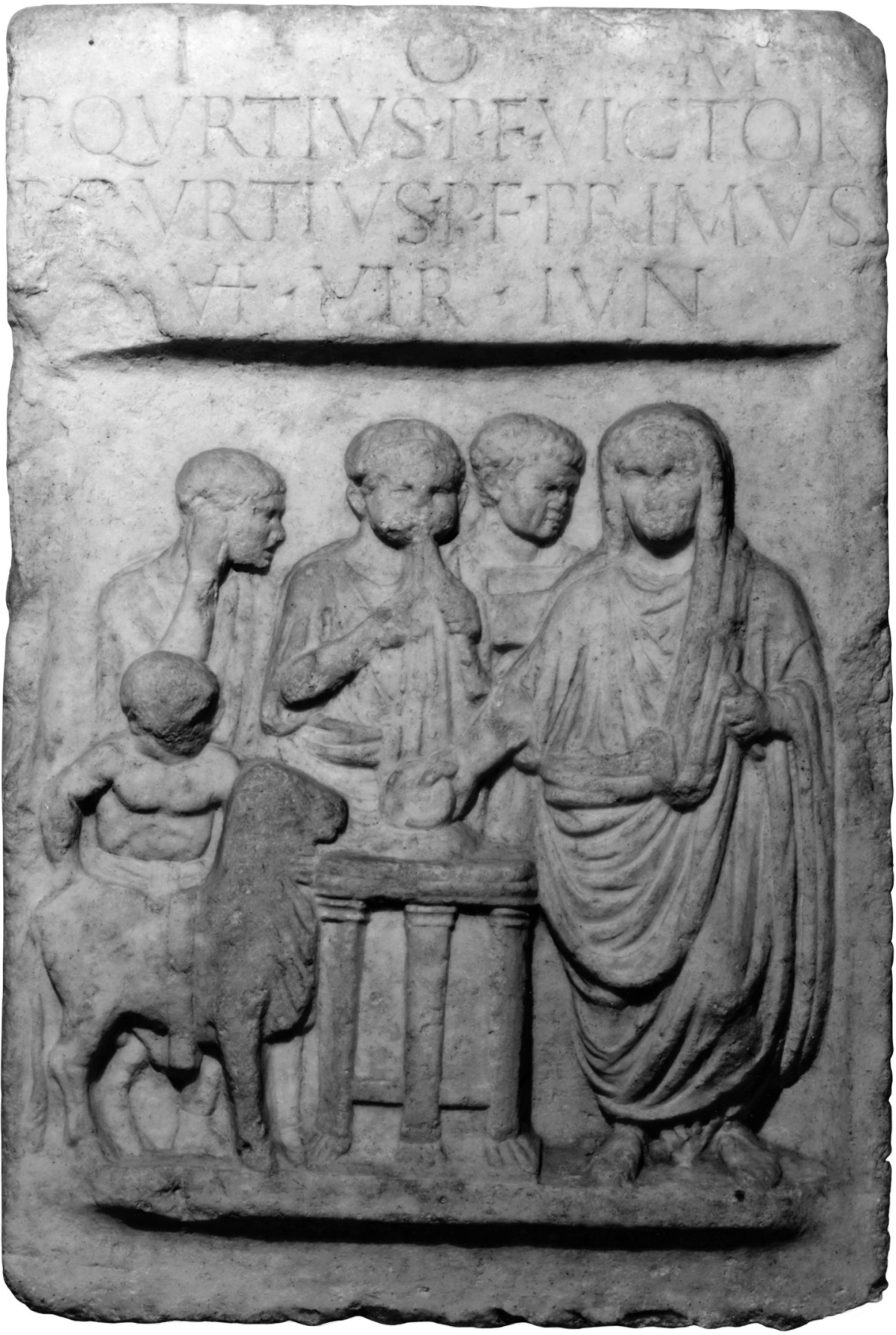


Qurtius Primus, probablement des frères, le monument représente le sacrifice dans la phase initiale de la praefatio ${ }^{102}$. La procession a conduit les participants près du trépied sur lequel le feu est allumé. Tout à gauche, derrière le desservant occupé à tenir le bœuf bientôt offert au dieu, un homme en toge est représenté de profil ${ }^{103}$. Son visage est tourné vers le trépied, sa bouche entrouverte - c'est le seul de la scène -, sa main droite portée à l'oreille. L'identification de l'individu n'est pas évidente et deux hypothèses sont possibles : il s'agirait soit du monitor, chargé de lire la formule exacte que devait prononcer le sacrifiant, soit du praeco, chargé d'appeler au silence rituel par un retentissant fauete linguis ${ }^{104}$. L'absence de représentation d'un rouleau dans la main du personnage tend à exclure la première solution, de même que son éloignement physique avec le sacrifiant: il s'agit de codes iconographiques que les sculpteurs semblent avoir développés pour représenter celui qui était garant du formulaire ${ }^{105}$. Par sa bouche ouverte et sa main obturant son pavillon auditif, l'individu en question paraît tout au contraire représenter le praeco qui prononçait, au nom du sacrifiant, l'appel au silence ${ }^{106}$. L'acte de transmission du message comme son contenu sont ici figurés.

Alors que le praeco appelle au silence, le sacrifiant, représenté tout à droite, est en train de renverser sa patère sur les flammes afin d'appeler Jupiter au sacrifice ${ }^{107}$. Un pan de sa toge est relevé sur sa tête: il pratique donc le sacrifice capite velato «à la romaine ${ }^{108}$ ». Cette particularité cultuelle a été relevée par Plutarque dans la dixième de ses Questions romaines: «Pourquoi, lorsqu'ils se prosternent devant les

102 - Corpus Inscriptionum Latinarum, V, 5472:I(oui) O(ptimo) M(aximo) $\mid P($ ublius) Qurtius P(ubli) f(ilius) Victor $\mid P($ ublius $) Q($ urtius $)$ P(ubli) f(ilius) Primus $\mid$ VIuir iun(ior) : «À Jupiter très bon très grand, Publius Qurtius Victor, fils de Publius, Publius Qurtius Primus, fils de Publius, sévir junior».

103 - Sur les desservants du culte et leur représentation dans l'art romain, outre le toujours utile Inez Scott RyberG, Rites of the State Religion in Roman Art, Rome, American Academy in Rome, 1955, voir Friederike FLEss, Opferdiener und Kultmusiker auf stadtrömischen historischen Reliefs. Untersuchungen zur Ikonographie, Funktion und Benennung, Mayence, Zabern Verlag, 1995.

104 - L'appel au silence n'était pas prononcé directement par le sacrifiant mais par un praeco, voir FESTUS, $78 \mathrm{~L}$ : Nam praecones clamantes populum sacrificiis fauere iubebant.

105 - Voir, par exemple, dans I. S. RYBERG, Rites of the State Religion ..., op. cit., les représentations sur le relief de Clodius Gothicus (pl. 67, fig. 116e), le relief sacrificiel provenant de Reggio di Calabria (pl. 67, fig. 116d), l'autel de Manlius (pl. 25, fig. 39a) ou l'une des scènes de sacrifice de la colonne Aurélienne (John Scheid et Valérie Huet (dir.), La colonne Aurélienne. Autour de la colonne Aurélienne. Geste et image sur la colonne de Marc Aurèle à Rome, Turhout, Brepols, 2000, p. 341, fig. 48, p. 227-242).

106 - Sur les fonctions des praecones et les conséquences sociales du fait de prêter sa voix à un magistrat, voir Jean-Michel DAVID, "Le prix de la voix: remarques sur la clause d'exclusion des praecones de la table d'Héraclée », in T. Hantos (dir.), Laurea Internationalis. Festschrift für Jochen Bleicken zum 75. Geburstag, Stuttgart, Franz Steiner, 2003, p. 81-106, ou encore Jean-Michel DAvid, «La baguette et la voix », in M. T. SCHETTINO et S. PitTia (dir.), Les sons du pouvoir..., op. cit., p. 313-327.

107 - La concentration de plusieurs étapes en un même temps de représentation est un phénomène courant et bien connu des images antiques. 
dieux, se couvrent-ils la tête, tandis que, lorsqu'ils rencontrent des hommes dignes de considération, ils se découvrent si précisément ils se trouvent avoir leur toge sur la tête? » L'hypothèse la plus plausible présentée par le polygraphe est la suivante: «Or ils se prosternaient ainsi devant les dieux ou en se cachant la tête par modestie, ou plutôt ils relevaient leur toge jusqu'aux oreilles, prenant garde que ne tombât sur eux de l'extérieur, pendant leurs prières, une parole de mauvaise augure et défavorable ${ }^{109}$. En relevant un pan de toge sur sa tête le sacrifiant créait donc un voile qui visait à le détacher de son environnement immédiat, non seulement visuel, en concentrant sa vue devant lui, mais aussi acoustique, par la mise en place d'un obstacle contre les mauvaises paroles ${ }^{110}$. Il s'agit de la deuxième stratégie destinée à créer un environnement propice, c'est-à-dire silencieux.

Le dernier agent du silence est représenté immédiatement derrière le trépied: les joues gonflées par l'effort, le regard concentré sur le foyer, un tibicen souffle dans ses tuyaux. Le joueur de tibia, cet instrument à hanche double presque toujours improprement traduit par «flûte» dans les éditions de textes modernes, est de loin le musicien que les sources associent le plus fréquemment aux rites de la religion publique ${ }^{111}$. Là où un regard anachronique pourrait ne voir dans la mise en musique du rite qu'un simple ornement, un embellissement liturgique, il convient au contraire de penser le jeu de la tibia comme un élément particulièrement important de la constitution du silentium rituel. Pline l'Ancien explique très clairement les stratégies et les finalités de cette purification de l'environnement sonore:

pour éviter qu'aucun des termes ne soit sauté ou interverti, quelqu'un lit d'abord la formule d'après le texte, tandis qu'un autre surveillant est placé pour en contrôler l'exactitude et qu'un troisième enfin est commis à faire observer le silence; pendant ce temps le tibicen se fait entendre pour qu'aucune parole ne puisse être perçue, deux faits remarquables ayant été retenus: chaque fois que des imprécations ont troublé le sacrifice ou que la prière a été mal faite, aussitôt la tête des entrailles ou le cœur ont disparu ou ont été trouvés doubles sans que la victime eut bougé ${ }^{112}$.

Les sources permettent donc d'appréhender un système élaboré, destiné à créer autour du principal acteur du sacrifice un environnement propice, le silentium. Le

109 - Plutarque, Étiologies romaines, 10.

110 - Sur le voile comme condition de réalisation orthopraxique du rite, voir Valérie HuET, «Le voile du sacrifiant à Rome sur les reliefs romains: une norme?», in F. GHERCHANOC et V. Huet (dir.), Vêtements antiques. S'habiller, se déshabiller dans les mondes anciens, Arles, Errance, 2012, p. 47-62.

111 - Alexandre VINCENT, Jouer pour la cité. Une histoire sociale et politique des musiciens professionnels de l'Occident romain, Rome, École française de Rome, 2016, p. 141-171.

112 - Pline L'ANCIEN, Histoire naturelle, 28, 11 : ne quod uerborum praetereatur aut praeposterum dicatur, de scripto praeire aliquem rursusque alium custodem dari qui adtendat, alium uero praeponi qui fauere linguis iubeat, tibicinem canere ne quid aliiud exaudiatur utraque memoria insigni, quotiens ipsae dirae obstrepentes nocuerint quotiensue precatio errauit; sic repente extis adimi capita uel corda aut geminari uictima stante. 
cri du praeco, le voile et le jeu du tibicen cherchaient à isoler le sacrifiant du cadre l'environnant selon un système de cercles concentriques de plus en plus efficaces. Le pan de la toge rabattu sur la tête n'offrait qu'une protection minime et symbolique contre les possibles paroles néfastes car il ne pouvait véritablement protéger les oreilles du sacrifiant des sons extérieurs. La voix du praeco élargissait la sphère sacrée dans la mesure où elle ne s'appliquait pas à un seul individu. L'espace couvert par la voix du héraut dépassait le cercle des plus proches desservants du sacrifiant. Son cri se voulait universel, il appelait tous les citoyens à ne pas perturber la communication en train de s'établir entre le responsable du sacrifice et les dieux: retenez vos langues, vous tous qui assistez à la scène! Il souffrait néanmoins d'une limite inhérente à sa nature même: cri unique, puissant mais réduit à deux mots, fauete linguis, sa valeur performative était restreinte dans l'espace et le temps. Rien ne garantissait le sacrifiant que ce silence favorable serait respecté aussi longtemps que le réclamerait le rite : les effets de sa parole étaient intrinsèquement volatils. La troisième modalité d'obtention du silence, le jeu de la tibia, offrait cette durée et cette ampleur qui manquaient au cri du praeco. Le tibicen pouvait jouer aussi fort et aussi longtemps qu'il était nécessaire, autant qu'il le faudrait pour l'achèvement parfait du rite. Instrument au corps d'os, d'ivoire ou de bois, la tibia permettait l'obtention de sons puissants, rendant matériellement crédible une isolation phonique entre le sacrifiant et le monde extérieur ${ }^{113}$. Pour cette raison, il faut supposer que le tibicen jouait par-dessus ces formules qui n'étaient pas destinées à être entendues par un hypothétique public. Le rôle du musicien était complémentaire de celui du praeco dans la mesure où tous deux cherchaient à annuler l'effet d'éventuelles paroles négatives perturbant les formules prononcées par le sacrifiant. Le héraut découpait un espace destiné au silentium rituel, que le musicien amplifiait grâce à son instrument. Contrairement au silentium augural, le silence requis par les sacrifices était donc, d'un point de vue acoustique, un silence empli de sons, autrement dit un silence bruyant.

Au sein d'une période chronologique relativement restreinte (la fin de la République et les premières décennies du Principat) dans le cadre de la religion publique romaine, le silence nécessaire à la bonne réalisation des rites a donc trouvé deux manifestations radicalement différentes: le silence parfait lors des prises d'auspices et le silence bruyant des sacrifices. Pour tenter d'expliquer cette variation pragmatique, il faut revenir aux fonctions assignées aux deux rituels. La prise d'auspices comme le sacrifice se définissaient comme des tentatives d'interaction entre le monde des dieux et celui des citoyens; ils étaient des temps de la communication, néanmoins leurs modalités variaient. La finalité de la prise

113 - L'idée selon laquelle les tibiae, plus particulièrement vouées aux cultes publics, aient pu être réalisées en ivoire dérive d'un passage de VIRGILE, Géorgiques, 2, 193 : cum pinguis ebur Tyrrhenus ad aras lancibus. On note toutefois qu'il s'agit d'un passage consacré aux cultes dionysiaques. La précision est aussi donnée par ProperCE, Élégies, 4, 6. Contra, Pline L'ANCIEN, Histoire naturelle, 16, 66, fait état, en son temps, de tibiae jouées dans les cultes et réalisées en buis: Nunc sacerdotae Tuscorum e buxo, ludicrae uero et loto ossibusque asininis et argento funt. Les vestiges archéologiques montrent une variété de matériaux possibles. 
d'auspices était l'obtention de l'assentiment des divinités, que ce soit sur le nom du magistrat nouvellement élu, la nécessité de mener bataille ou encore l'opportunité d'ouvrir une assemblée populaire. Il s'agissait donc d'un rituel de communication dans lequel l'expressivité, la "parole", revenait aux dieux. Or à l'exception de Numa, dans le célèbre passage des Fastes où le roi demande à Jupiter d'expliquer les raisons de son mécontentement malgré les réformes entreprises dans la cité, aucun être historique, aucun homme, n'a jamais parlé avec les dieux ${ }^{114}$. Les dieux ne s'exprimaient pas directement, ils n'utilisaient pas le langage des humains; en d'autres termes, au regard des hommes, les dieux étaient muets ${ }^{115}$. Là où le langage participait à la définition de l'humanité, les dieux étaient caractérisés par le silence ${ }^{116}$. Le silentium augural dépassait donc le simple cadre pragmatique propice à la réception des signa visuels ou sonores d'expression des dieux: il était le signe de l'assentiment divin. La manifestation divine, si elle advenait, ne pourrait qu'être synonyme du désaccord de la divinité, qu'il importait dès lors de limiter, d'où la dimension mécanique du rite décrit par le passage cicéronien ${ }^{117}$. La communication via le sacrifice correspondait à un processus inverse à celui de la prise d'auspices: il n'était pas question d'écouter les dieux mais il s'agissait de s'adresser à eux, de leur parler, et de leur parler tel que le prescrivaient les traditions religieuses. La protection de la parole du sacrifiant contre toutes celles qui pourraient venir le perturber, contre les dirae obstrepentes, pour reprendre l'expression de Pline l'Ancien, primait sur tout. Le sacrifiant, agissant au nom de la cité, se protégeait donc par la mise en place d'un paravent sonore dont la fonction était de masquer la parole humaine.

Y a-t-il une réalité plus immédiatement compréhensible que le silence? Défini par ce qu'il n'est pas (le bruit, la voix, le discours...), le silence se donne a priori comme une donnée naturelle immédiatement appréhensible par tous, à tel point que l'idée même d'en écrire une histoire pourrait surprendre. La récente entreprise d'A. Corbin est donc louable, comme toute tentative de compréhension analytique des phénomènes sociaux. Car le silence est tout le contraire d'une donnée naturelle. Fruit d'une perception et d'une définition contextualisées, appréhendé par des sens culturellement, socialement et politiquement formés, le silence est soumis, comme les autres perceptions sensorielles, à interprétation et variation. Néanmoins, en contraignant l'étude aux émotions suscitées par le silence, l'auteur limite son ampleur. L'ouverture à d'autres sources et la considération du silence comme catégorie sonore, faisant partie d'un plus vaste paysage sonore, permet d'en libérer la richesse. La notion inventée par M. Schafer, précisée par une

114 - Ovide, Fastes, 3, 260-392. Pour la bibliographie concernant ce passage abondamment commenté, voir F. PRESCENDI, Décrire et comprendre le sacrifice..., op. cit., p. 189-198. 115 - Annie Dubourdieu, «Divinités de la parole, divinités du silence dans la Rome antique ", Revue de l'histoire des religions, 220-3, 2003, p. 259-282, ici p. 260 sq. 116 - Corrado BoLOGNA, «Il linguaggio del silenzio. L'alterità linguistica nelle religioni del mondo classico », Studi storico-religiosi, 2-2, 1978, p. 305-342, ici p. 317. 
définition fondée sur les acquis récents de l'histoire et de l'anthropologie des sens, ouvre des perspectives de comparaison. La recherche de la signification du silentium religieux dans l'Antiquité romaine offre un cas d'étude précis. Derrière une apparence d'uniformité nominaliste, ce silence est composé de réalités acoustiques nettement différenciées qui forment autant d'appels à la nuance et à des études contextualisées fines, combinant lexicographie, enquête historique et démarche anthropologique.

Alexandre Vincent

Université de Poitiers, HeRMA EA 3811 